\title{
Molecular Dynamics Simulation of Polysulfone and Polystyrene-co-maleic Anhydride Blends Compatibility: A mesoscopic, Ewald Approach and Experimental Comparison
}

John Michael Tesha ( $\sim$ jcollinstesha@hotmail.com )

Tiangong University

\section{Research Article}

Keywords: Blend-module simulation, Polysulfone, Poly (Styrene-co-maleic Anhydride), DSC analysis, Phase Diagram, Energy of mixing

Posted Date: December 10th, 2021

DOI: https://doi.org/10.21203/rs.3.rs-1109742/v1

License: (c) (1) This work is licensed under a Creative Commons Attribution 4.0 International License. Read Full License 
Molecular Dynamics Simulation of Polysulfone and Polystyrene-co-maleic Anhydride Blends Compatibility: A mesoscopic, Ewald Approach and Experimental Comparison

\author{
John Michael Tesha ${ }^{a, b, c *}$
}

${ }^{a}$ State Key Laboratory of Separation Membranes and Membrane Processes, National Centre for International Joint Research on Separation Membranes, Tiangong University, Tianjin 300387, P. R. China

${ }^{b}$ School of Materials Science and Engineering, Tiangong University, Tianjin 300387, P. R. China

${ }^{c}$ Tanzania Bureau of Standards, P.O. Box 9524, Dar es salaam, Tanzania

*Corresponding authors: john.tesha@tbs.go.tz / jcollinstesha@hotmail.com (Dr Tesha J.M.) 
23 Graphical Abstract:

24

25



26 
28

29

30

31

32

33 34

35 36

Amorphous cells built to perform MD simulations NVT-300 ps protocol at various blending ratios

Schneier theory and thermodynamics study shows that PSF and PSMA is thermodynamically compatible The simulation reveals the PSF/PSMA blend is miscible at T higher than $400 \mathrm{~K}$ PSMA 
Abstract:

This work aims to use molecular modeling to envisage the compatibility of Polysulfone (PSF) and Poly (styrene-co-maleic anhydride) (PSMA) polymers blend. A blend-module was developed based on the molecular dynamics (MD) technique compared to an experimental study. Molecular dynamics simulations were achieved using the condensed phase-optimized molecular-potentials for atomistic simulation studies (COMPASS) force field with atomic-based electrostatic. The PSF/PSMA blend compatibility facets and thermodynamic Gibb's free energy across ranges of PSF/PSMA blend compositions were calculated. In doing so, the Flory Huggins chi interaction parameter of mixing $(\chi)$ and solubility parameters $(\delta)$ were computed from $298 \mathrm{~K}$ and on increasing temperature to predict the miscibility of the polymers blend in the amorphous cell model by atomistic simulations. It was found that the blendsystem is miscible using the interaction chi parameter of Florry Huggins at a temperature above 400K. At higher time-step, mesoscopic simulations for PSF/PSMA reached equilibrium and computed free energy. Mixing energy indicated the stability of the PSF/PSMA polymer blend. The results of this work narrate to the Flory Huggins theory enthalpy of mixing for binary blend polymers at 40 and $60 \%$ PSMA. Additionally, the kinetic phase of the miscibility/immiscibility of the PSF/PSMA blend system's miscibility/immiscibility was examined using Differential Scanning Calorimetry (DSC). The result confirms the good interaction between the two polymers through the shift of glass transition temperature ( $\mathrm{Tg}$ ) values within individual polymers $\mathrm{Tg}$. It is crucial to investigate the miscibility of two different polymers for a variety of polymer applications. The MD simulation provides a powerful, accurate computational tool in the estimation of polymer compatibilities. 
Keyword: Blend-module simulation; Polysulfone; Poly (Styrene-co-maleic

63 Anhydride); DSC analysis; Phase Diagram; Energy of mixing

64 


\section{Introduction}

Different types of polymer blends have been studied to make new polymer material, such as polymeric membranes, which depend on the polymer-polymer blending's miscibility [1]. There is increasing attention to studying different polymers with their numerous applications in recent years [ㄹ, $\underline{3}]$. Studies indicated that Molecular Dynamics (MD) simulations are essential tools, which can avoid countless experimental and human errors in predicting the miscibility or blend compatibility of polymers. $[\underline{4}, \underline{5}][\underline{6-8}] .$. In conjunction with MD simulations, Flory-Huggins theory allows the easy way to study the compatibility of the blend polymers. MD simulations have complex systems type simulations that enable the interactions between bonded and non-bonded atoms for the built correct polymers $[\underline{9}, \underline{10}]$. The interactions can be combined in forcefield files in Material Studio. The simulations can be run using Condensed-phase Optimized Molecular Potentials for atomistic simulations studies (COMPASS), the techniques confirmed by various researchers [ㅁ, 11-13]. The simulation systems are very sensitive to parameters set in the forcefield environment. Among the properties of polymers, the interaction parameters can be estimated from numerical paths of the blend polymers, which contain monomers' statistical structure properties to mimic the polymers' behavior $[\underline{14}, \underline{15}]$.

Herein Polysulfone (PSF) common known polymer and has been studied extensively in membrane applications due to its stability and potential in growing membrane technologies. It has been broadly applicable in the area such as ultrafiltration, microfiltration, and its surface modification for nanofiltration and reverses osmosis for water treatment [16]. The modification of PSF can be done by blending with other polymers. However, it is essential to be aware of compatibility or incompatibility to achieve successful blending [17-19]. Poly(styrene-co-maleic anhydride) (PSMA) 
versatile copolymer used membrane technology through blending with other polymers to achieve attractive membranes with desirable features such as antifouling, hydrophilicity, and heavy metals rejection and compatibility of PSMA and with polymers has the great technological and scientific interest to researchers to achieve successful fabrication of membranes [20-22].

This study aims to predict the compatibility/incompatibility of the PSF and PSMA by molecular dynamics simulations - forcite blending tool in material studio 2017 software, and comparison with experiments such as thermal properties of these polymers (Differential Scanning Calorimetry (DSC) and thermostability thermogravimetric analysis - (TGA)). Furthermore, this present study reports the MD simulation results obtained from the Discover Amorphous cell module of the material studio 2017. The results obtained using simulations in conjunction with FloryHuggins theory allows us to foretell the phase behavior of PSF/PSMA blending at various compositions. Thermodynamics analysis and using Schneier's approach of the equation were as well employed to envisage thermodynamic compatibility. 


\section{Theoretical relations}

107 MD simulations were done with the commercial software Material Studio (2017) 108 from Accelrys Inc. San Diego, CA, USA. The molecular mechanics and dynamics 109 simulation in the discover module and building of amorphous cells were done in this work. The geometric optimization and potential energy estimations of arbitrary periodic and molecular systems using classical mechanics were done with the forcite module's help. Optimization is done to achieve the most stable structural configuration. This optimization is conducted in two-steps in the system,

114 conformation adjustment and energy evaluations.

115 The blend-module using Dreiding [23] forcefield was performed in this work. The 116 "Dreiding" forcefield exemplifies intermolecular and intramolecular interaction 117 between polymers. The amorphous cell construction module was done in this work 118 with simulations giving the ability to study molecular systems and poly material type 119 by predicting the model's critical properties. This algorithm is suitable for building 120 longer chains and molecules in two separate steps, first creating an initial guess 121 structure and second structural relaxation to the minimum potential energy state [24 , 122 25]. In this study, Condensed-phase Optimized Molecular Potentials for Atomistic 123 Simulation Studies (COMPASS) forcefield was employed since it allows accurate 124 prediction of the molecules' properties in the broad range, such as conformation, 125 structural vibrational, and thermos-physical properties. These properties are in 126 isolated condensed-phases in a wide range of conditions of pressure and temperature.

127 This is a consistent polymer forcefield (PCFF) used in modeling and simulation for 128 atomistic interaction, and the potential energy total of the system, is the result of the 129 sum of non-bond, bond (valence) and cross term interaction energies as shown in Eq. 130 (1) 


$$
E_{\text {TOT }}=E_{\text {valence }}+E_{\text {crossterm }}+E_{\text {non-bond }}
$$

The valence interactions energy ( $\left.E_{\text {valence }}\right)$ consists of energies of the bond stretching $\left(\mathrm{E}_{\mathrm{Bond}}\right)$, valence angle bending energy $\left(\mathrm{E}_{\text {Angle }}\right)$, dihedral angle torsion energy $\left(\mathrm{E}_{\text {torsion }}\right)$, and out of plane energy interaction or energy inversion ( $E_{\text {oop }}$ or $\left.E_{\text {inversion }}\right)$. Modern COMPASS forcefield has a new denomination known as Urey Bradley ( $\left.\mathrm{E}_{\mathrm{UB}}\right)$ that takes into account the interaction between pairs of atom in 1 to 3 configurations that is atom bonded to a common atom. Eq. (2) used in the system to obtain Evalence.

$$
E_{\text {valence }}=E_{\text {Bond }}+E_{\text {torsion }}+E_{\text {Angle }}+E_{\text {oop }}+E_{U B}
$$

The forcefield's accuracy is done by introducing the correction factor as a bond or angle distortion caused to the nearby atom. This is determined in cross-term interaction energy $\left(\mathrm{E}_{\text {crossterm}}\right)$. This term gives out the dynamic properties of the molecule through vibration frequencies, and later the interaction energy between non bonded atoms ( $\left.E_{\text {non-bond }}\right)$ consists of the energy of Van der Waals $\left(E_{\mathrm{vdw}}\right)$, coulomb energy electrostatic ( $\left.E_{\text {coulomb }}\right)$, and energy of Hydrogen bond ( $\left.E_{H-b o n d}\right)$ shown in Eq. (3)

$$
E_{\text {non-bond }}=E_{H-\text { Bond }}+E_{\text {Coulomb }}+E_{v d w}
$$

In COMPASS, Van der Waals's energy $\left(E_{\mathrm{vdw}}\right)$ is defined by the electrostatic and potential energy from partial atom charges in a system calculated by the charge equilibration method [26]. The electrostatic interaction was estimated by the Ewald summation method, which is highly accurate in the estimation of long-range molecular interaction [27]. The geometric optimization was done on each polymer PSF, and PSMA and the forcite module were used for this optimization at 5000 steps of energy minimization. The tolerance convergence for force and energy is 0.005 $\mathrm{kcal} / \mathrm{mol} 1 \times 10^{-4} \mathrm{kcal} / \mathrm{mol}$, respectively. 


\subsection{Blends tool calculation and simulation}

\subsubsection{Parameters to predict the miscibility}

The strength between molecules of the polymer materials is defined by the solubility parameter (The Hilderbrand) $-\delta[\underline{28}]$, which results in blending miscibility. This parameter is calculated by the square root of cohesive energy density (CED), as shown in Eq. (4)

$$
\delta=\sqrt{\mathrm{CED}}
$$

The important aspect of the simulation is the polymer molecular weight. Selecting the length of a polymer chain in individual PSF and PSMA polymers, MD simulation was used by performing dynamics to estimate $\delta$. The stable value of $\delta$ is obtained at sufficient individual monomers repeating units for running the simulation [29].

In this study, atactic PSF of 20 repeating unit monomer (Fig. 2) and isotactic alternate copolymer PSMA of 39 repeating unit monomer (Fig. 2) used to construct the amorphous cell weight fraction ratio $1: 1$ of the blend. Varying the repeating units from 20-80 allows us to build the cell at different weight fraction ratios for the blend. The polymers' densities were employed from the literature study [30], where PSF density set $1.24 \mathrm{~g} / \mathrm{cm}^{3}$ and PSMA density set as $1.08 \mathrm{~g} / \mathrm{cm}^{3}$, and such a system gives out 945 atoms to be investigated for 1:1 polymer repeat unit blend. Fig. 1 shows the schematic procedure of simulation used to develop the polymer blends and interactions that illustrate the methodology. 


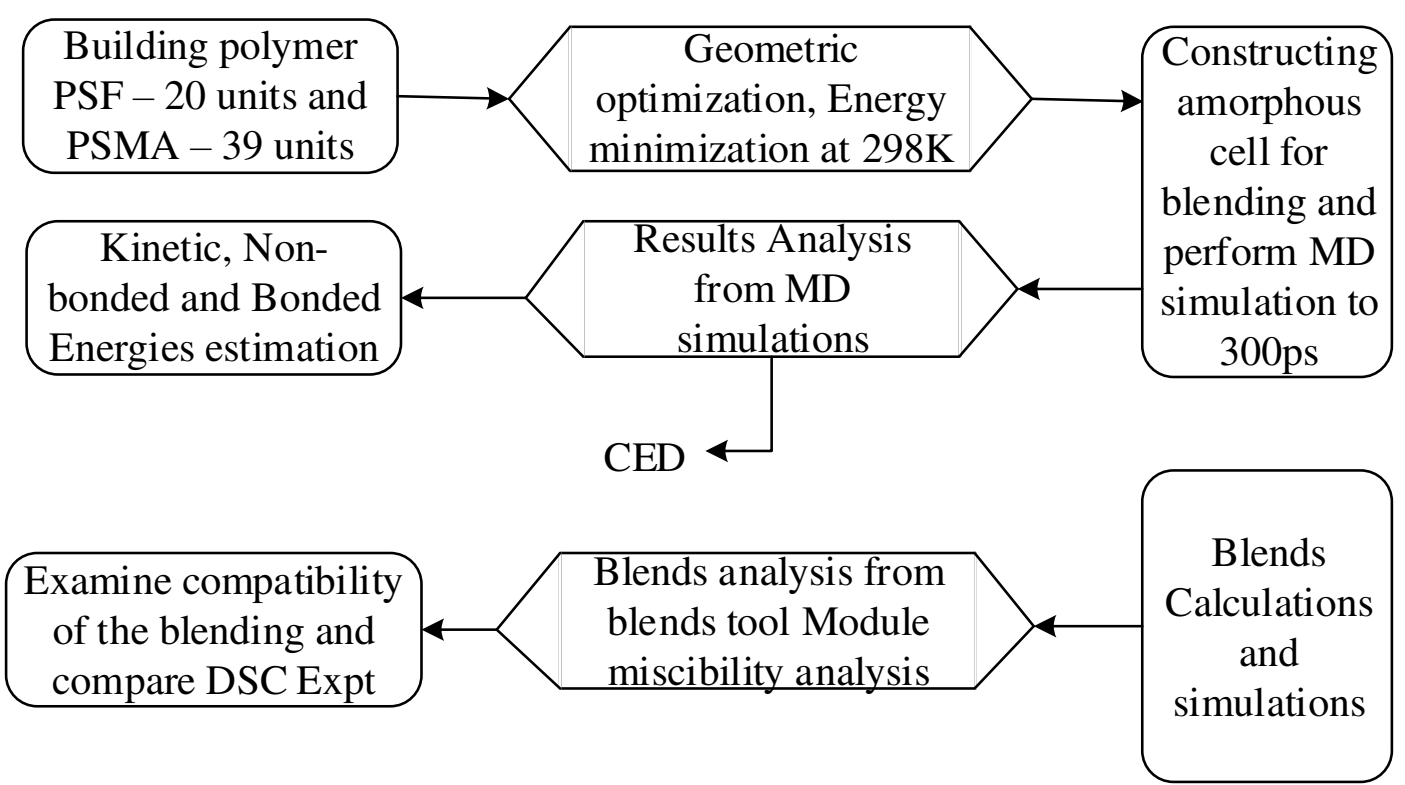

175

Fig. 1: Schematic procedure for simulation to develop the structure for polymer blends

Polymer molecular weight does not vary with cohesive energy density (CED). However, it is highly related to the interaction of the polymer, and by neglecting intramolecular forces, CED increases with energy $\left(\mathrm{E}_{\mathrm{coh}}\right)$ per mole $(\mathrm{V})$ of the polymer shown in Eq. (5). The solubility parameter values are shown in Table 1, comparing literature and calculated from the forcite tool - CED.

$$
\therefore C E D=\frac{E_{c o h}}{V}
$$

It was reported that the CED depends on conversion degree and temperature to achieve the solubility parameter. This shows that there is a linear relationship between crosslinking density and CED. Therefore, the increase in temperature results in a decrease in the solubility parameter, and the temperature has many effects with a polymer having a lesser conversion degree [31].

Thermoset polymer with a higher conversion degree has a lower cohesive energy density and solubility parameter. The cohesive energy density shows a linearly decreasing relationship with the crossslink density. These properties also decrease with increasing temperature and in thermoset polymers with lower conversion degrees show a more significant temperature effect. 


\begin{tabular}{cccccc}
\hline Polymer & $\begin{array}{c}\text { Repeating } \\
\text { Unit }\end{array}$ & $\begin{array}{c}\text { Molecular weight } \\
\text { repeating unit }(\mathrm{g} / \mathrm{mol})\end{array}$ & $\begin{array}{c}\text { Density } \\
\left(\mathrm{g} / \mathrm{cm}^{3}\right)\end{array}$ & $\begin{array}{c}\text { Solubility parameter }\left(\mathrm{cal} / \mathrm{cm}^{3}\right)^{0.5} \\
\text { COMPASS }\end{array}$ \\
\hline PSF $^{\mathrm{a}}$ & 20 & 442.5 & 1.238 & 8.27 & Experimental \\
\hline PSMA $^{\mathrm{b}}$ & 39 & 202.1 & 1.079 & 8.01 & $9.92[\underline{30}]$ \\
\hline
\end{tabular}

195 Different researchers have reported other value solubility parameters from the PSF

196 experiment [33-35] and PSMA [36-39]. For this reason, it is important to take into

197 consideration the accurate method of using MD simulation for obtaining interaction

198 between these polymer blends depending on their CED. The blend-module in

199 simulation in predicting miscibility has a superior unique high efficiency taking into

200 account the Flory-Huggins interaction parameter $(\chi)$ across a range of temperatures

201 and ideal conditions. This method can envisage the thermodynamics of mixing

202 directly from two polymers' chemical structures (PSF and PSMA). It requires the

203 input of these structures into the blends simulation. This type of simulations'

204 uniqueness depends on combining the modified Flory-Huggins modeling [40-42] with

205 the MD simulation system [43].

206 The CED computation can enable us to obtain the energy of mixing $\left(\Delta \mathrm{E}_{\mathrm{mix}}\right)$ since the

207 Flory-Huggins model of this binary system is based on the thermodynamics of mixing

208 of the polymer/polymer system.

209 Generally, the energy of mixing is expressed, as shown in Eq. (6)

$$
\Delta E_{\text {mix }}=\Phi_{a}\left(\frac{E_{c o h}}{V}\right)_{a}+\Phi_{b}\left(\frac{E_{c o h}}{V}\right)_{b}-\left(\frac{E_{c o h}}{V}\right)_{\text {mix }}
$$

211 The terms that are in brackets represent cohesive energies of individual pure polymers

$212(\mathrm{a}, \mathrm{b})$ and the polymer blend (mix). However, $\Phi_{\mathrm{a}}$ and $\Phi_{\mathrm{b}}$ are volume fractions of each

213 polymer in the blending system. The equation 
expressed in terms of free energy mixing, as shown in Eq. (7)

$$
\frac{\Delta G}{R T}=\frac{\Phi_{a}}{n_{a}} \ln \Phi_{a}+\frac{\Phi_{b}}{n_{b}} \ln \Phi_{b}+\chi \Phi_{a} \Phi_{b}
$$

Whereby $\Delta \mathrm{G}$ is the free energy of mixing (per mole), $\mathrm{T}$ is the reference absolute temperature of the simulation (in Kelvin), $\mathrm{R}$ is the molar gas constant, and $\chi$ is the Flory-Huggins interaction parameter. The beginning two terms in equation Eq. (7) Are called combinatorial entropy, this is always negative, thus favoring a mixing state in the pure polymers. Finally, in the last term, the interaction parameter (FloryHuggins) can be expressed according to case F.H [44], as shown in Eq. (8). When the parameter is positive, the combinatorial entropy disfavors the polymer blend's mixing state and balances these two terms in Eq. (7), resulting in different phase diagrams.

$$
\chi=\left(\frac{\Delta E_{m i x}}{R T \Phi_{a} \Phi_{b}}\right) V
$$

Generally, this interaction parameter is essential in indicating the polymer miscibility, and it obeys the critical value, as shown in Eq. (9).

$$
\left(\chi_{a b}\right)_{\text {critical }}=\frac{1}{2}\left(\frac{1}{\sqrt{m_{a}}}+\frac{1}{\sqrt{m_{b}}}\right)^{2}
$$

Whereby $m_{b}$ and $m_{a}$ are the degrees of polymerization of individual pure polymer. Therefore, when the blend system's interaction parameter is lower than the $\left(\chi_{\mathrm{ab}}\right)_{\text {critical }}$, then the system is miscible for the whole polymer composition range. Likewise, if the blend system's interaction parameter is slightly higher than the critical value, then the system exhibits the partial miscibility of the polymer blend. Besides, the two phases can exist, containing both compositions. Therefore, for significantly higher interaction 
parameter values $(\chi)$ than critical, the system becomes completely immiscible. By comparing the values of $(\chi)$ estimated from the equation Eq. (9) with the atomistic simulation, the compatibility or miscibility behavior of the polymer blends can be predicted $[\underline{45}, \underline{46}]$. The traditional Flory-Huggins modeling, each component in the blend system, has the lattice site with coordination number $\mathrm{Z}$ in which the energy mixing is given by equation Eq. (10)

$$
\Delta E_{m i x}=\frac{1}{2} Z\left(E_{s b}+E_{b s}-E_{b b}-E_{s s}\right)
$$

Ebb is the binding energy base-base pair, Ebs is a binding energy base-screen pair, $\mathrm{E}_{\mathrm{sb}}$ is the binding energy screen-base pair, and $\mathrm{E}_{\mathrm{ss}}$ is the binding energy screen-screen pair. These are energies of interaction between two polymers and, together with the interaction parameter $(\chi)$, can generate energy mixing.

\section{The blends-button is selected from the menu toolbar and then the menu's dropdown to} select calculation from the module dropdown list. In the setup input section tab, the empty row under the molecule must be assigned to input the structures that have been geometrically optimized by forcite. In the Blends dialogue calculation setup, the quality accuracy has been changed from medium to fine, corresponds to a bin width of 0.2 $\mathrm{kcal} / \mathrm{mol}$ to $0.02 \mathrm{kcal} / \mathrm{mol}$, respectively. After that, it uses the same dialogue (Blends Calculation) energy tab selected for setup. The forcefield was set to Dreiding and changed the charges to the 'charge using ' $\mathrm{QEq}$ ' to calculate the Flory-Huggins interactions. Finally, the Run option was selected in the blends calculation dialogue to achieve the polymer's mixing. During the blending simulation, the new file of the PSF mixture was created in Project Explorer. After the simulation was completed, the Blend-Module analysis was selected from the menu bar. The analysis dialogue in the blend-module allows us to examine the interaction parameter $(\chi)$, the energy mixing, and the phase 
diagrams. This blend-module system was validated by its calculations done in the Material studio $[\underline{47}, \underline{48}]$.

\subsubsection{Experimental solubility parameters}

\subsubsection{Schneier theory and Thermodynamics analysis}

The enthalpy of mixing $\Delta m$ reported from Schneier's theory equation Eq. (11) can also determine the compatibility of the polymers using experimental parameters from different literature and depending on the density of each polymer $\rho_{a}$, and $\rho_{b}$ the solubility parameter of each polymer $\delta_{a}$, and $\delta_{b}$ the molecular weight of each polymer $\mathrm{M}_{\mathrm{a}}$ and $\mathrm{M}_{\mathrm{b}}$ and weight fraction for each polymer $\mathrm{W}_{\mathrm{a}}$ and $\mathrm{W}_{\mathrm{b}}$. The subscript components "a" and "b" represent two polymers blend, and for this report, specifically, "a" is PSF, and "b" is PSMA. The Schneier equation below is used to obtain the enthalpy of mixing for blended polymers, of which from the calculations, it was reported by Flory-Huggin's theory that for the composite polymers to be compatible, the enthalpy of mixing $\Delta m<0.01 \mathrm{cal} / \mathrm{mol}$ likely for incompatible polymer blend the enthalpy of mixing $\Delta m>0.01 \mathrm{cal} / \mathrm{mol}$.

$$
\Delta \mathrm{H}_{m}=\left\{W_{a} M_{a} \rho_{a}\left(\delta_{a}-\delta_{b}\right)^{2}\left[\frac{W_{b}}{\left(1-W_{b}\right) \mathrm{M}_{b} \rho_{b}+\left(1-W_{a}\right) M_{a} \rho_{a}}\right]^{2}\right\}^{\frac{1}{2}}
$$

In order to obtain the status of thermodynamics compatibility, the Gibbs free energy $\Delta G_{m i x}$ of blended polymer can be used to determine the thermodynamics compatibility state. Gibbs free energy $\Delta G_{m i x}$ is dependent on the enthalpy of mixing $\Delta H_{m}$ of the mixed polymers calculated from the above equation, Entropy of mixing $\Delta S_{m i x}$, and temperature of mixing $\mathrm{T}(\mathrm{K})$ at which mixed polymers become miscible [49]. Different researchers have reported that Gibbs's free energy increase with the increase in compatibility degree. Therefore, the thermodynamics equation Eq. (12) is 
282 used to calculate the Gibbs free energy of mixing, and according to the 283 thermodynamics theory [50] for thermodynamic compatible polymers blend, the 284 Gibbs free energy $\Delta G_{m i x}<0 \mathrm{cal} / \mathrm{mol}$ and thermodynamic incompatible $\Delta G_{m i x}>0$ $285 \mathrm{cal} / \mathrm{mol}$.

$$
\Delta G_{m i x}=\Delta H_{m}-T \Delta S_{m i x}
$$




\subsection{Materials}

291 Polysulfone (PSF) molecular weight about 97,000 g/mole with repeating unit 292 molecular weight $442.52 \mathrm{~g} / \mathrm{mole}$. It was purchased from Dalian Polysulfone Plastic 293 Company Ltd (Liaoning province China), Crystal-clear powder form PSMA-5 294 molecular weight about $\left(1.2 \times 10^{5}\right) \mathrm{g} / \mathrm{mole}, \mathrm{MA}=26 \%$ wt was purchased from Solvay 295 solexis (Belgium).

296 Fig. 2 shows the monomers' chemical structure (PSF and PSMA) used in this study.

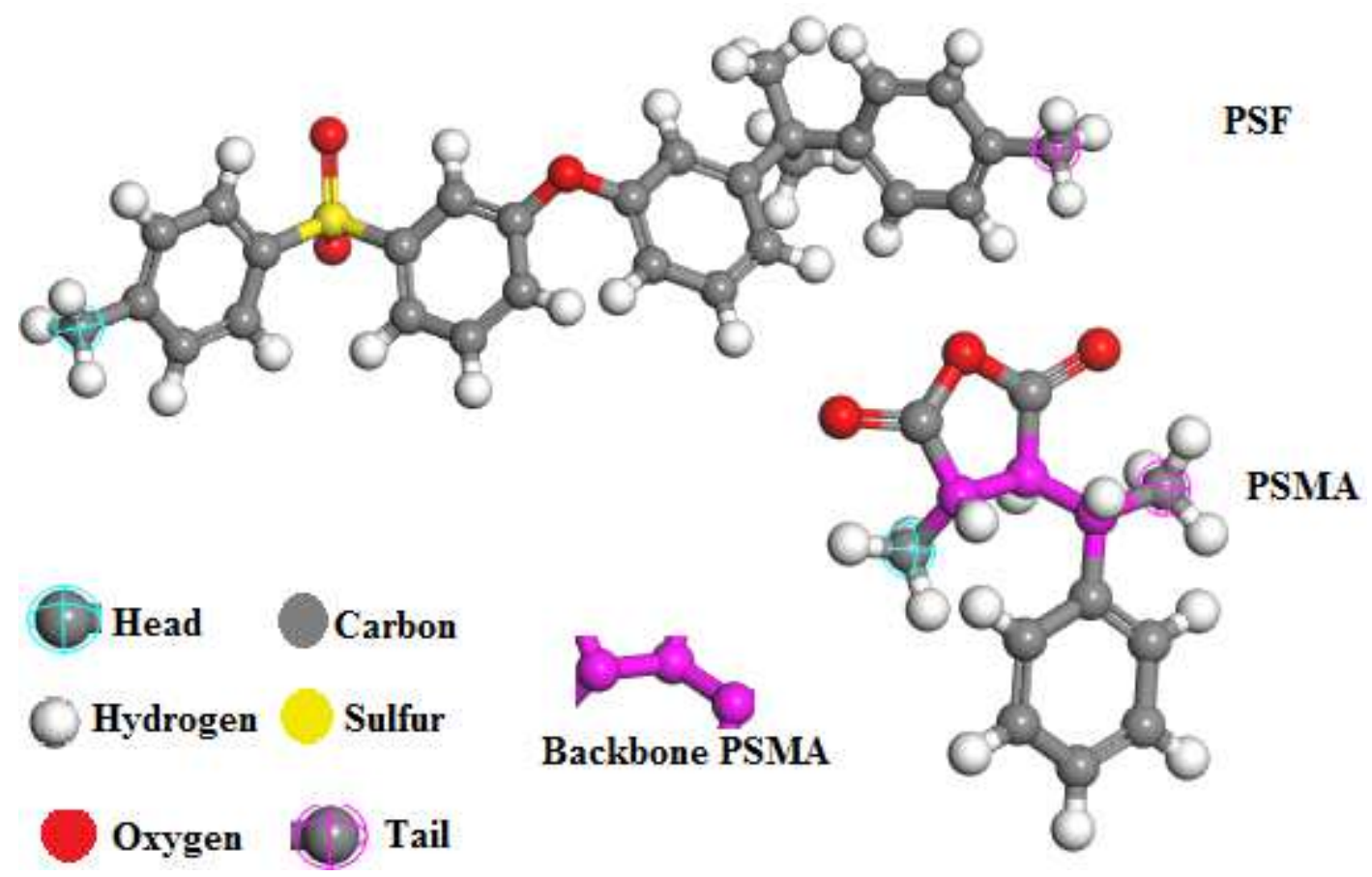




\subsection{Thermal Properties Analysis}

Table 2 indicates the denomination to describe the samples used in this study for thermal properties. The polymer miscibility criterion is to obtain a single glass transition temperature $\left(\mathrm{T}_{\mathrm{g}}\right)$ in a polymer blend. This is the result of the miscibility at the molecular level of the polymer blends. Additionally, the single $\mathrm{T}_{\mathrm{g}}$ can indicate the homogeneity of the polymer chain's distribution in polymer films [51].

Table 2: Description of the samples-polymer concentrations

\begin{tabular}{ccc}
\hline Name & PSF $(\% \mathrm{wt})$ & PSMA $(\% \mathrm{wt})$ \\
\hline T0 & 100 & 0 \\
T1 & 80 & 20 \\
T2 & 60 & 40 \\
T3 & 40 & 60 \\
T4 & 20 & 80 \\
T5 & 0 & 100 \\
\hline
\end{tabular}

\subsubsection{Differential Scanning calorimetry (DSC) investigation}

The glass transition temperature $\left(\mathrm{T}_{\mathrm{g}}\right)$ values of pure polymers and polymer blend samples were studied by NETZSCH DSC 200F3 Maia-Germany model instrument to examine the polymer blend and rigidity's miscibility of the polymers. Samples were measured at an average of $8-12 \mathrm{mg}$, and the thermal scanning was conducted from $20{ }^{\circ} \mathrm{C}$ to $220{ }^{\circ} \mathrm{C}$ at a heating rate of $10{ }^{\circ} \mathrm{C} / \mathrm{min}$ under the flow of nitrogen $\left(\mathrm{N}_{2}\right)$.

\subsubsection{Thermogravimetric Analysis (TGA)}

NETZSCH STA449-F3-Jupiter instrument was used to analyze the thermal stability of the polymers. Samples at different polymer concentrations were weighed at an average of 17-19 mg and transferred to Alumina ceramic crucible for thermal analysis. Crucibles are non-reusable with high-temperature stability, a wide range of operating 
327 [52]. TGA study was done from $40{ }^{\circ} \mathrm{C}$ to $800{ }^{\circ} \mathrm{C}$ at a constant heating rate of

$32820{ }^{\circ} \mathrm{C} / \mathrm{min}$, and nitrogen gas was used at a flow rate of $1 /(\mathrm{ml} / \mathrm{min}): 50$.

\section{4. Results and Discussion}

\subsection{DSC Results of PSF/PSMA}

331 One of the most typical experimental methods to evaluate the polymer-polymer 332 compatibility is by determining the polymer blend's Tg value and comparing it with 333 an individual pure polymer (PSF and PSMA). This method can experimentally study 334 the compatibility between the PSF and PSMA polymer. Fig. 3 shows the results of the DSC for the PSF/PSMA.
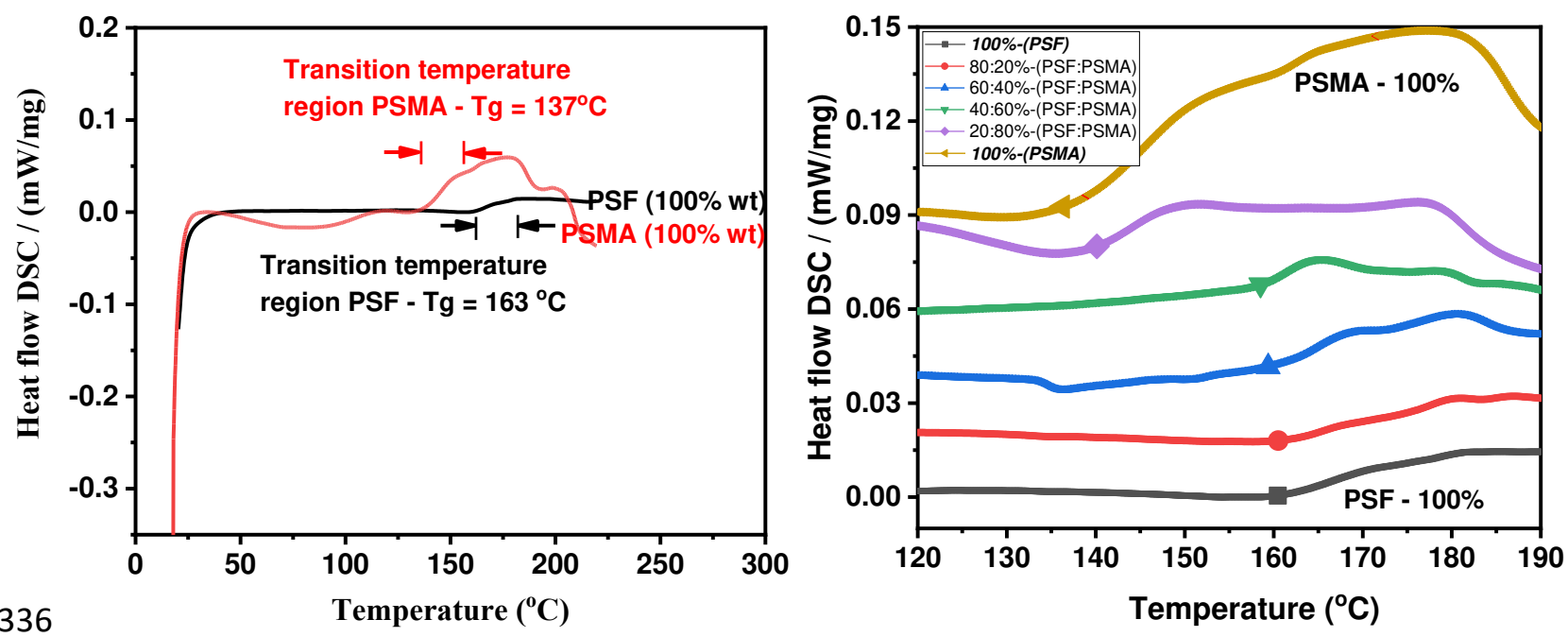

Fig. 3: DSC spectra of PSF/PSMA blending at various concentrations ratio

338 The criteria behind the polymer miscibility are obtaining the single value of $\mathrm{Tg}$ in a polymer blend system, which indicates the miscibility at the molecular state [51].

340 From Fig. 3 it is showing that the $T_{g}$ of individual polymers is different. That is, pure

341 PSF has a $\mathrm{T}_{\mathrm{g}}$ of $163{ }^{\circ} \mathrm{C}$, which is higher than pure PSMA having $\mathrm{T}_{\mathrm{g}}$ of $137{ }^{\circ} \mathrm{C}$.

342 However, varying polymer concentration of polymer as indicated in Table 2, the $\mathrm{T}_{\mathrm{g}}$

343 values change and different from that pure PSF and pure PSMA. These differences 
can be due to many van der Waals interactions between two polymers that lose the identity [53].

Furthermore, there is a modeling interaction as the blend's distinct Tg values are in between those of individual polymers (PSF and PSMA). As an increase in the PSF polymer fraction in the blending, $\mathrm{Tg}$ 's value increases uniformly due to the higher $\mathrm{Tg}$ of PSF. The miscibility is observed below 50\% of PSMA as it is seen from Fig. 3 that a single gradual increase of $\mathrm{T}_{\mathrm{g}}$ values approaches a similar value to that of PSF.

351 However, above $50 \%$ PSMA, the blend $\mathrm{T}_{\mathrm{g}}$ is seen to shift closer to pure PSMA and

352 become immiscible. These results suggest further analysis by MD simulation to 353 understand and compare the miscibility at the edge boundary to avoid overestimations 354 and obtain the equilibrium polymer-polymer interaction and miscibility boundary 355 [54].

\subsection{Thermogravimetric Analysis (TGA)}

357 The thermal analysis for the PSF/PSMA data collected from the TGA instrument is 358 indicated in Fig. 4.

361 For pure PSF, the degradation onset temperature is seen at $484.9{ }^{\circ} \mathbf{C}$, and thermal 362 degradation continued up to a temperature of $542.9{ }^{\circ} \mathbf{C}$. Conversely, pure PSMA is 363 seen to have a degradation onset temperature at $301.8{ }^{\circ} \mathbf{C}$, and the final degradation 364 temperature was found to be $\mathbf{4 2 8 . 8}{ }^{\circ} \mathbf{C}$. This is attributed to the chemistry nature of the 365 two polymer structures, where cleavage of $\mathrm{C}=\mathrm{C}$ in benzene (aromatic) ring in PSF 366 will be last, and $\mathrm{C}-\mathrm{O}$ in maleic anhydride cleaved more easily compared to $\mathrm{C}=\mathrm{C}$ of PSF $[\underline{55}, \underline{56}]$. The degradation behavior between pure PSF and pure PSMA continued to be seen (PSF/PSMA-polymer blend). The blending of PSMA in the PSF matrix improved thermal stability at a low concentration of PSMA up to $40 \%$ of the polymer 370 blend. The stability from 60:40\% (PSF:PSMA) blend up to 100\% pristine PSF was found to have an onset degradation temperature of $484.9{ }^{\circ} \mathrm{C}$ and a final degradation temperature of $550.8{ }^{\circ} \mathbf{C}$. This is attributed to the significant part of the blend occupied by highly thermally stable pristine PSF.

Additionally, a further increase of PSMA (20:80\%-PSF:PSMA) lowers the thermal stability, and degradation onset temperature was observed to decrease to $316.8{ }^{\circ} \mathbf{C}$, and final degradation temperature was found to be $414.5{ }^{\circ} \mathrm{C}$. It is evidently confirmed 377 that the two polymers' interaction at a low concentration of PSMA shows good agreement. As indicated in Fig. 4, a single degradation peak in the TGA graph. This 379 confirms the PSF/PSMA blend's miscibility is in the range of 0 to $40 \%$ PSMA, as 380 seen in the uniform behavior between the two pure polymers.

\section{$381 \quad 4.3 \quad$ MD Simulations}

382 Structure built in the Material Studio before running simulations was optimized at 3835000 steps. Fig. 5 is shown that profiles were generated during the optimization of 384 these structures. The optimization aims to bring the structures' stable configuration, 385 conformation, and minimal energy evaluation to be used for constructing amorphous cell and blending simulation. It is seen that PSF structure energy was minimized from 

reduced from $3603 \mathrm{kcal} / \mathrm{mol}$ to $-1578 \mathrm{kcal} / \mathrm{mol}$ using the conjugate gradient method.

389 This is also done in order to bring its excellent performance and reliability from the 390 initial starting point [57].
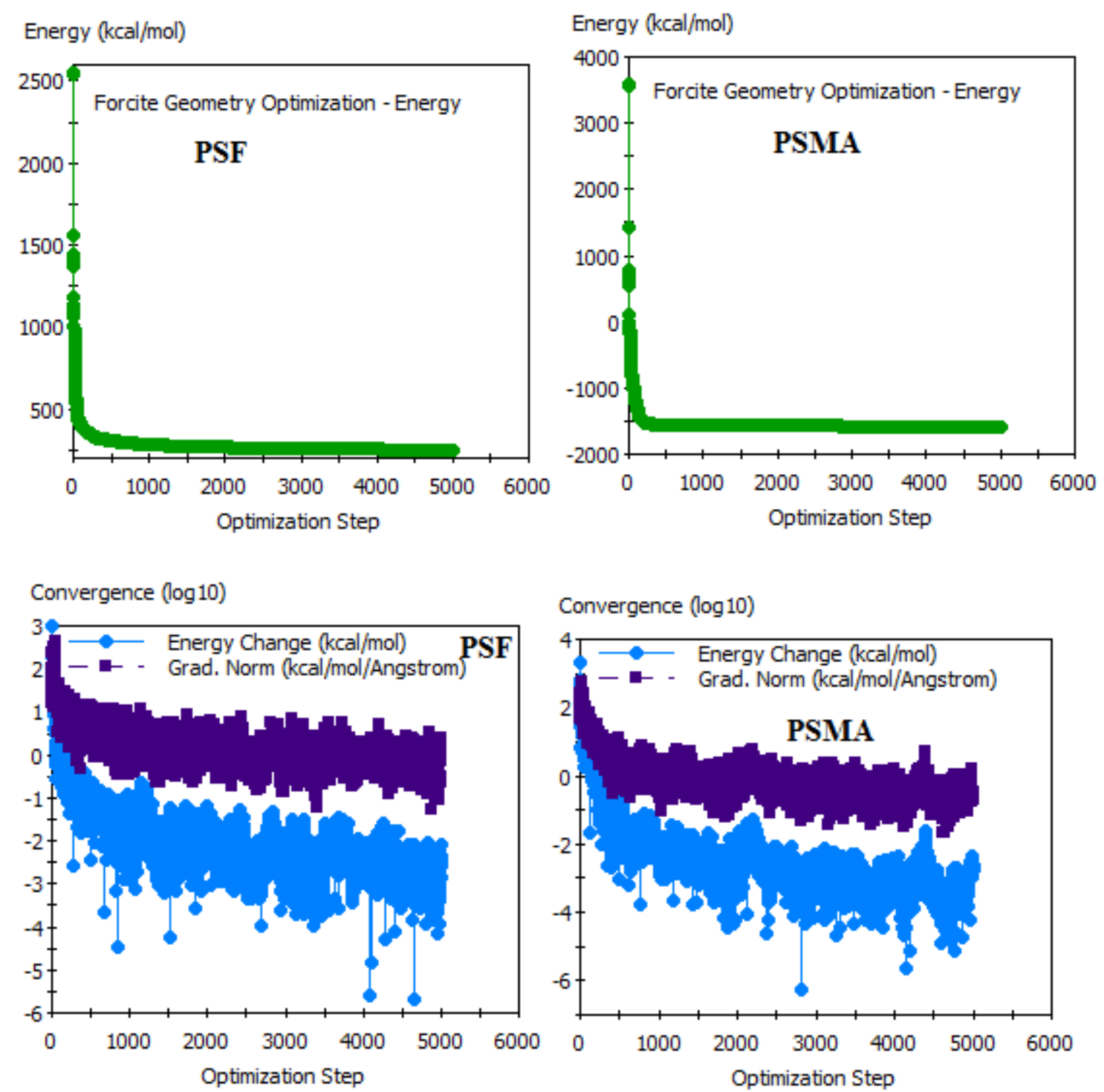

Fig. 5: Energies minimization and convergence profiles generated during optimization

\section{Amorphous cell construction and Dynamics analysis}

Unit cells of three-dimensional cubic were constructed having conditions of the

397 periodic boundary in amorphous cell module, as seen in Fig. 6. The cells were 398 optimized to minimize energy before running MD simulations. The simulation runs at $399300 \mathrm{ps}$, and $298 \mathrm{~K}$ with NVT ensemble means constant-volume/constant-pressure 
400 dynamics, this ensemble dynamics are set in such a way to allow the system to

401 exchange heat with the environment at the controllable temperature.
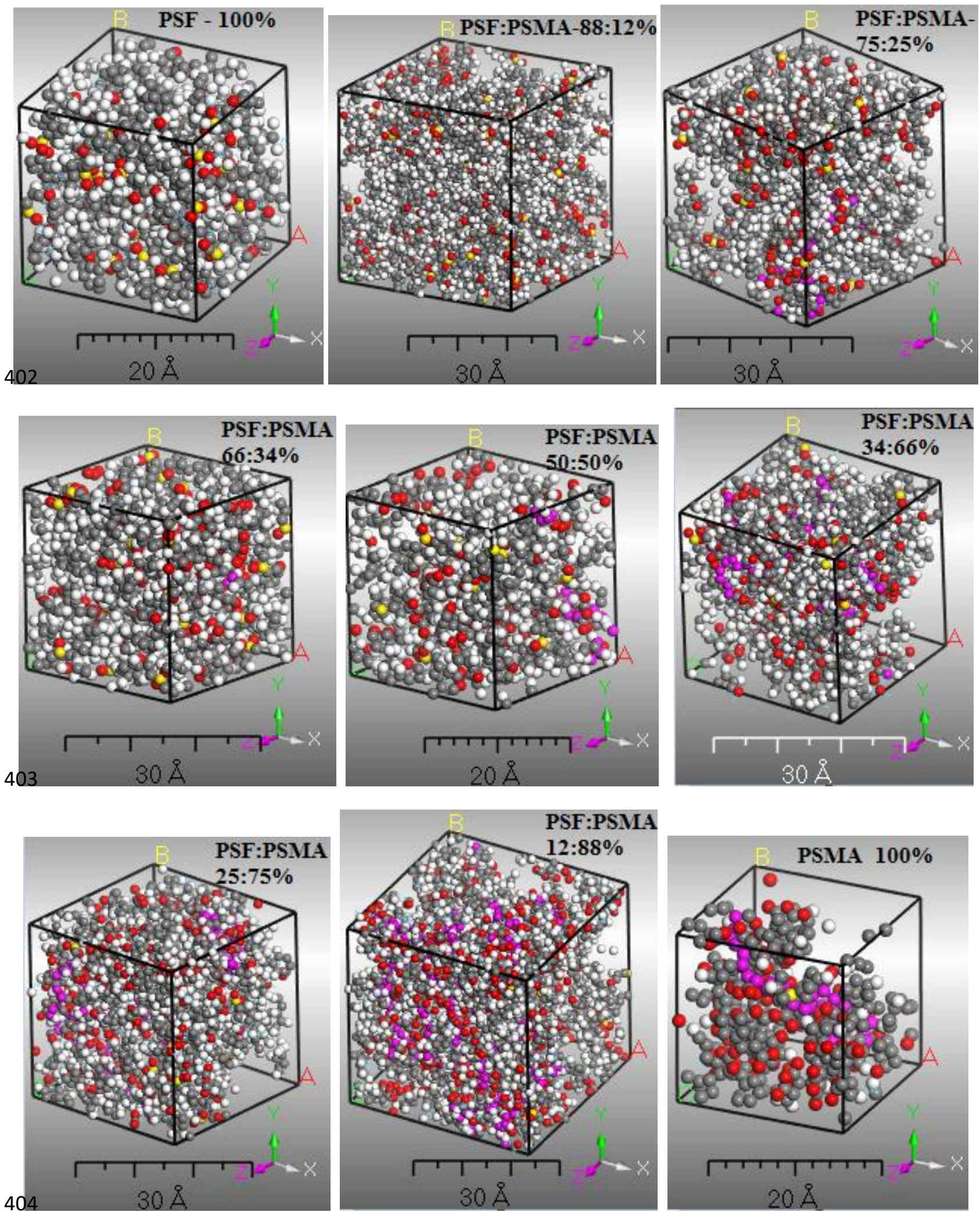
Here Carbon atoms are shown grey; Oxygen atom is shown red in color, Sulfur atoms

408 from PSF yellow in color, and Hydrogen are shown white. For model protocol,

409 different ratios number of chains are constructed in a unit cell. During MD simulation,

410 fluctuation profiles of temperature, non-bonded, potential, and total energies were

411 generated for each unit cell. Fig. 7 shows an individual pure polymer PSF and PSMA

412 generated profiles (temperature and energies). Further studies on each cell are shown

413 in Supplementary Figure S1
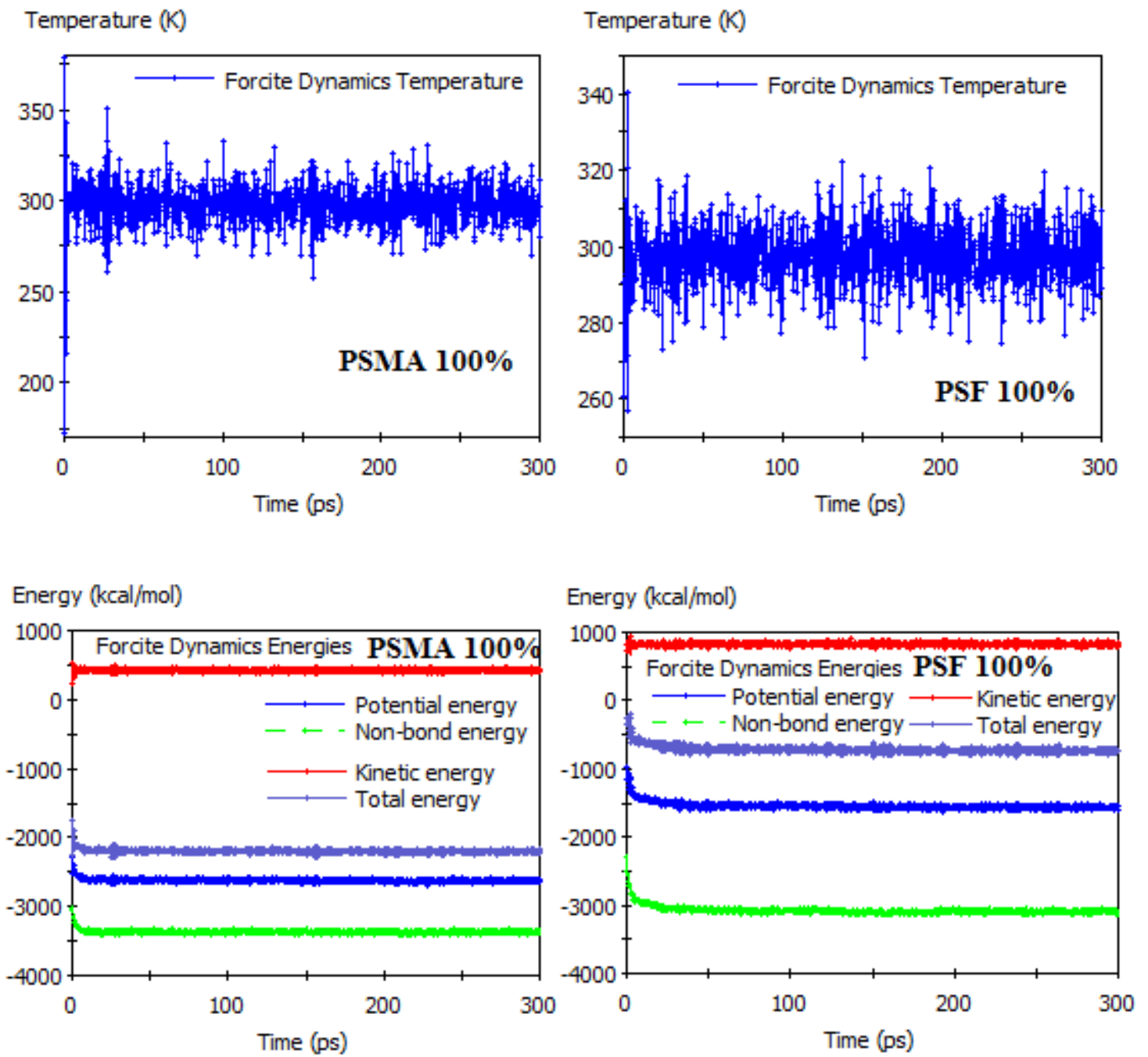
total energy plot vs. MD simulation time in ps. 
The results show that, for the duration of 300 ps simulation time, there is no significant change in the structures; hence, the time was adequate to attain the state of equilibration protocol. Also, to circumvent the entrapment of the simulated systems in a local metastable state of the minimized energy from geometric optimization, MD simulations were run at NVT ensemble then followed by NPT (constant pressure/content temperature dynamics) so to overcome the energy barrier between the minimal high energy by thermal energy provided [ 6$]$. The projected density of each amorphous cell is assumed from $1.24 \mathrm{~g} / \mathrm{cc}$ to $1.08 \mathrm{~g} / \mathrm{cc}$ from individual polymers.

\subsection{Flory-Huggins interaction parameter from blend-module analysis}

According to the theory of Flory-Huggins, when the interaction parameter $(\chi)$ is less than one and up to negative values, two molecules at the particular temperature are miscible, or interaction is favored. Furthermore, if $\chi$ is a positive value or higher than one, it indicates that two molecules can not be easily mixed $[\underline{40}, \underline{41}]$. Likewise, when the interaction parameter value is substantial, the free energy overcomes the combinatorial entropy of mixing, and the molecules will separate into two different phases. This part aims to obtain the value of $\chi$ for our two polymers blend (PSF and PSMA). However, the material studio software was capable of producing plots, as indicated in Fig. 8 from the Blend-module analysis dialogue at various temperatures to predict miscibility. The $\chi$ value obtained at $298 \mathrm{~K}$ particular temperature was 5.014, which confirm immiscible. However, from this result, it shows that the interaction parameter changes continue to change on the increase in temperature where the system becomes miscible from $400 \mathrm{~K}$ temperature, and above, at this particular temperature $(400 \mathrm{~K})$, the Flory-Huggins interaction parameter was seen to shift to lower values than 1 up to negative values. 

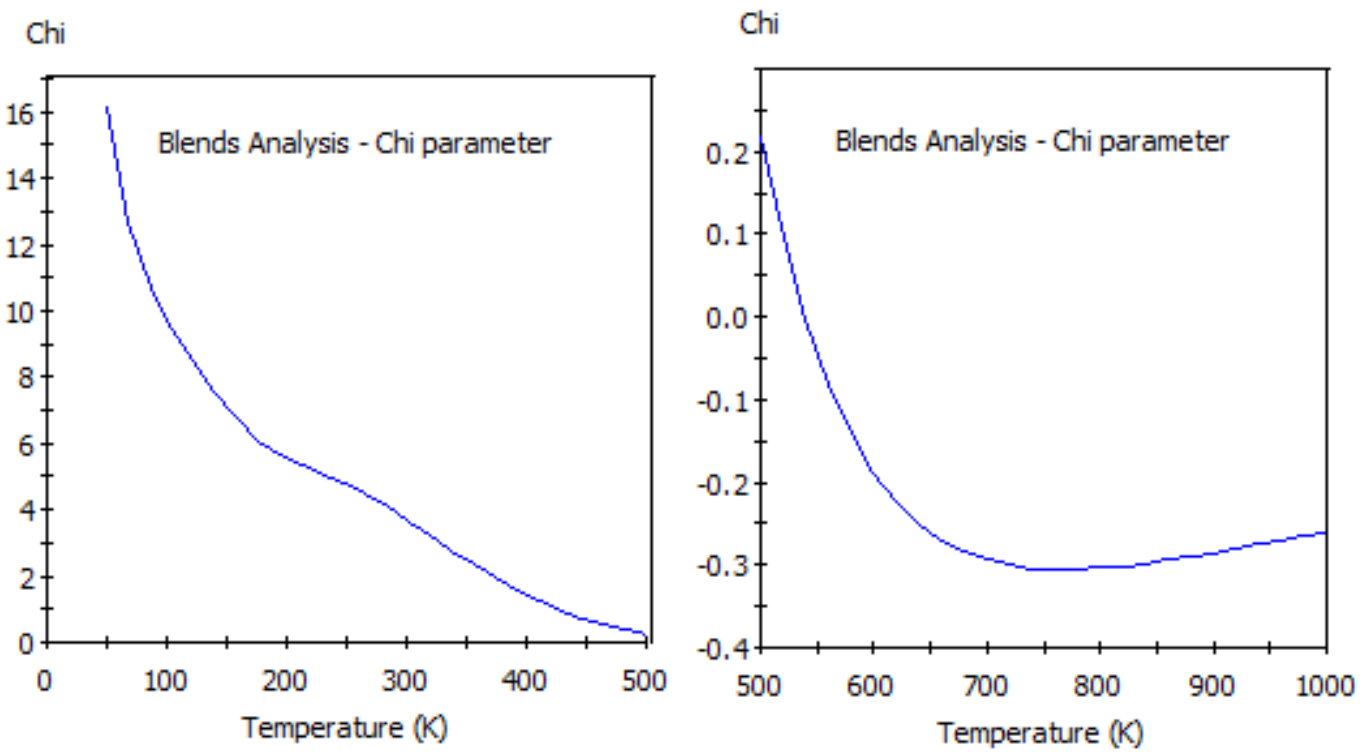

443

444

445

446

447

448

449

450

451

452

453

454

455

456 457 simulations. parameter.

Fig. 8: Flory-Huggins interaction parameter $(\chi)$ as a function of temperature (K) for PSF and PSMA polymers blending

Blends combines a modified Flory-Huggins model and molecular simulation techniques to calculate the compatibility of binary mixtures. Two important extensions to the Flory-Huggins model are employed:

Blends incorporates an explicit temperature dependence on the interaction parameter. This is accomplished by generating a large number of pair configurations and calculating the binding energies, followed by temperature averaging the results using the Boltzmann factor and calculating the temperature-dependent interaction

Blends is an off-lattice calculation, meaning that molecules are not arranged on a regular lattice as in the original Flory-Huggins theory. The coordination number is explicitly calculated for each of the possible molecular pairs using molecular in the blend simulation. 
Also, by substituting the temperature-dependent interaction parameter, $\chi$, in the Flory-

461 Huggins expression, the free energy is known for all compositions and temperatures.

462 From this, the phase diagram of the mixture can then be determined by locating the 463 critical point, the coexisting curve (binodal), and the stability curve (spinodal) in the 464 two-phase diagram.

465

471 simulation

As with any molecular simulation, the results obtained depend on the accuracy of the forcefield. Blends supports a wide variety of forcefields.

In the traditional Flory-Huggins model, each component occupies a lattice site. Although it is possible to use a fixed lattice coordination number in Blends, in calculating the interaction parameter $\chi$, Blends also provides the option to calculate the coordination numbers for each pair and considered when running blending simulation

472 In addition to the interaction parameter, it is crucial to analyze the system's mixing 473 energy and free energy to observe the value close to zero, suggesting miscibility. 474 Figure S2 can be used to confirm further on miscibility study of PSF and PSMA. The more decrease of energy mixing to less than zero (negative values) indicates PSF and 476 PSMA are miscible. The free energy plot at the various temperature as a function of 477 the mole fraction of PSMA was generated from the system's analysis. This plot is 478 presented in Fig. 9. This plot can be further analyzed using the enthalpy of mixing by 479 Schneier's theory (at 298K), which will be discussed later in this study. However, as 480 the temperature increases, it is free to shift to the negative side at the similar 481 composition of PSMA. This is attributed to the temperature-dependent miscibility 482 behavior, as confirmed earlier in this work. 

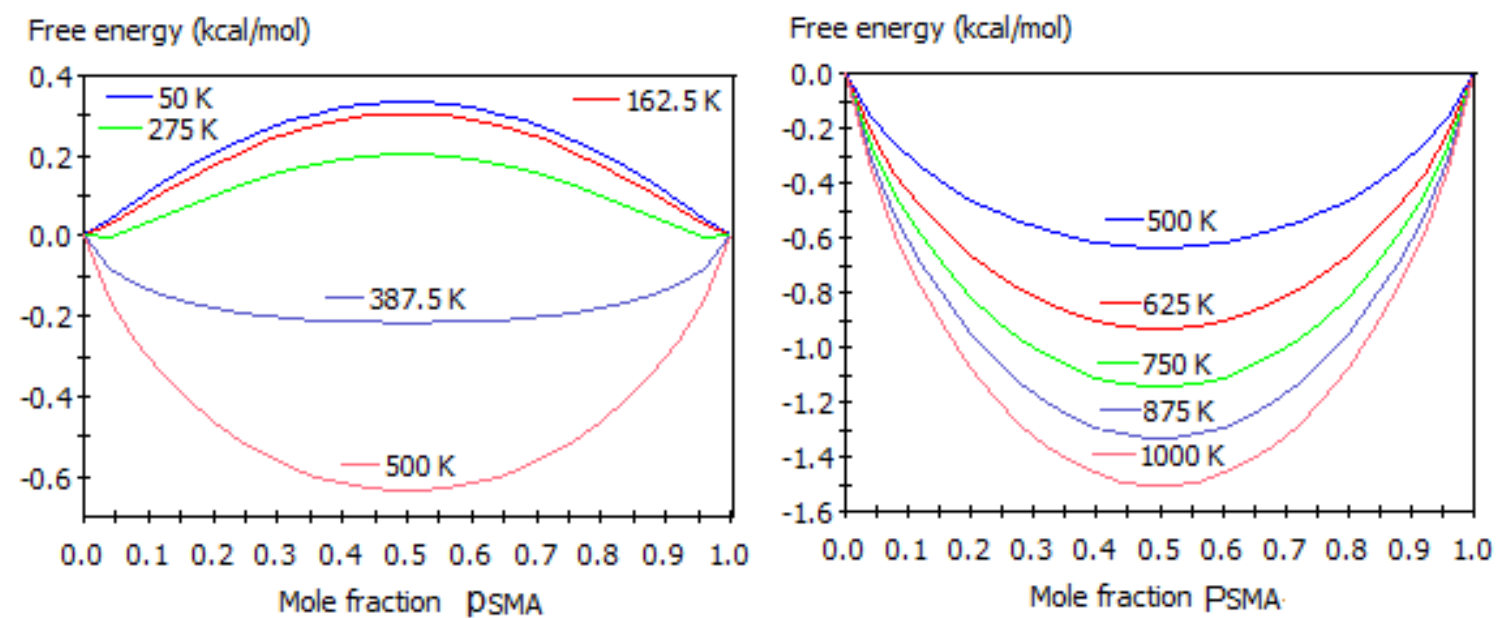

Fig. 9: Free Energy analysis function of mole fraction of PSMA

\subsection{Phase Diagram of the PSF/PSMA blend}

In illustrating the compatibility between polymers, phase diagrams are essential as range of PSF/PSMA mixture for miscible and immiscible. An immiscible polymer mixture splits into two phases, wherein the phase diagram, and we can read its composition. Fig. 10 is the phase diagram obtained from the blend-module analysis.

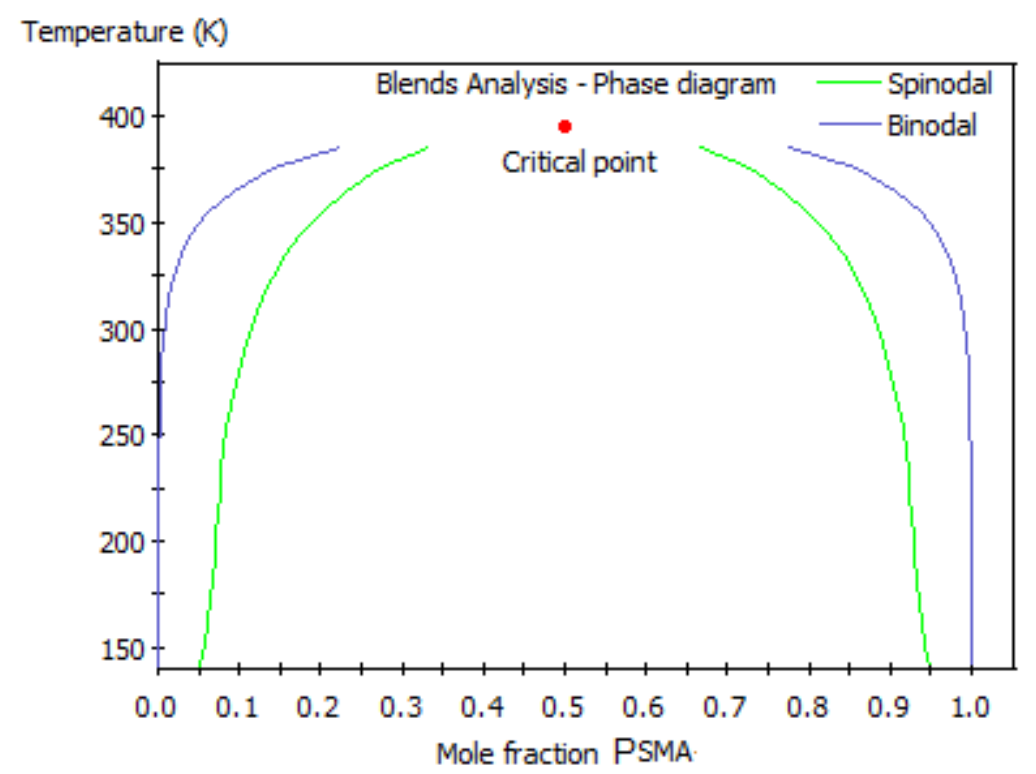


The pieces of information obtained from this plot, such as critical point (in red dot), Binodal (Blue line color), and spinodal (Green line color). Furthermore, these results show that at the critical point, there is a coexistence region for PSF / PSMA and the PSF/PSMA blend by split into two phases, which results in lower free energy, as illustrated earlier. The temperature at a critical point found was $400 \mathrm{~K}$ at a mole fraction of 0.5 PSMA. This plot has a single critical point that confirms one coexistence region. However, a complex binary system of polymer blend may have more than one critical point in its phase diagram due to several coexistence regions. Thus, the spinodal is linked to the coexistence region's separation into two phases, whereas the binodal links with the coexistence region in this phase diagram. In the region between the spinodal and binodal, the polymer blend system is metastable. That is, the blend will separate only after sufficiently high fluctuation. The region in the spinodal bounded up to lower temperatures the PSF/PSMA is immiscible, which agrees from the earlier illustration of Flory Huggins interaction parameter and mixing energy. Any fluctuation causes immiscibility as the system is unstable in the spinodal region. Additionally, above the binodal region, PSF /PSMA blend system is miscible and stable.

Recent advances in molecular simulation techniques have improved this situation. Accurate forcefields can be obtained by defining parameters using structural and spectral data. Molecular simulations can be performed on well-characterized systems, leading to a better fundamental understanding of atomic-level interactions. This information can then be used to predict useful physical properties of systems that are less well characterized. Many factors govern mixing processes, including the temperature and the chemical nature of the individual components. Additional factors may be involved for polymers; for example, chain packing, the degree of crystallinity, molecular weight, and chain flexibility. Not all of these factors can be addressed using 
molecular simulation techniques. However, it is possible to obtain structural data for

521 the individual components in a mixture and to calculate the interaction energy terms

522 required for the thermodynamic expressions.

523

\subsection{Compatibility by Schneier theory and thermodynamics}

525 Solubility parameters from the experimental work together with densities and 526 molecular weight of the repeating units can be used to estimate enthalpy of mixing 527 using Eq. 11. Therefore, the enthalpy of mixing obtained using experimental data of 528 solubility parameters from Table 2 can be plotted against the mole fraction of PSMA, 529 as indicated in Fig. 11. It is mentioned that the Schneier theory of compatibility is 530 assumed only at the temperature of the blend system $298 \mathrm{~K}$, and interest lies in the 531 system of compatible solids at room temperature. Hence solubility parameters and 532 density used in the calculation were at 298K [60]. Therefore the result disagrees with 533 simulations; however, only at $40 \%$ up to $60 \%$ PSMA blend agree with MD 534 simulation blends. Only $60 \%$ of PSMA agree with the DSC experiment, and below 40\% PSMA as illustrated earlier in this study.
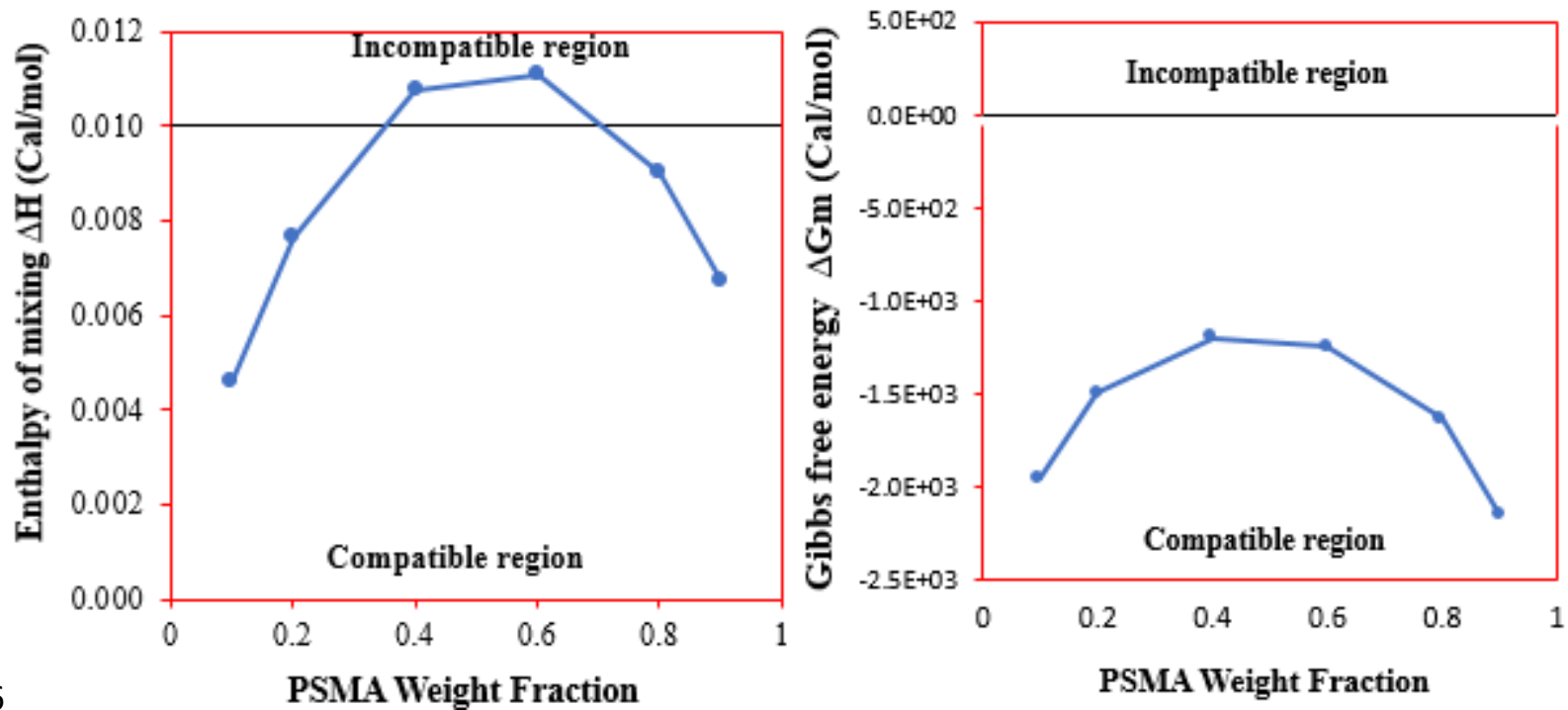
540 It is seen that the PSF/PSMA polymer blend is partially compatible. From 0.4 to 0.6

541 PSMA wt/wt, the enthalpy of mixing is higher than the limit of Flory Huggin's theory

$542\left(\mathbf{1 0}^{-3} \mathbf{~ c a l} / \mathbf{m o l}\right)$, which changes to the incompatibility on increase further PSMA weight

543 fraction turn to the compatible state. Therefore, from the Schneier equation

544 calculation, the system is partially compatible, which allows further investigation,

545 such as MD Simulations, compared to the experimental DSC approach. In Fig. 11.

546 From these $\mathbf{( 1 2}$, it is seen that Gibb's free energy is dependent on the enthalpy of

547 mixing calculated from the Schneier equation, the entropy of mixing and temperature

548 at which polymer blend system, higher temperature $>298 \mathrm{~K}$ as higher than $400 \mathrm{~K}$ the 549 system is thermodynamically compatible with an increase pf PSMA polymer 550 composition in the blend system.

551 5. Conclusion

552 In this study, the miscibility between PSF and PSMA was intensively examined by 553 MD simulation and the experiment. The energy mixing, Flory-Huggins interaction 554 parameters, amorphous cell, and phase diagrams were studied in this work. The 555 results showed that PSF and PSMA are miscible from a temperature above 400K with 556 an optimal weight fraction of PSMA of about 0.5. In the DSC experiment, the two 557 polymers show a good interaction as the shift of $\mathrm{T}_{\mathrm{g}}$ values within the range of 558 individual polymer $\mathrm{T}_{\mathrm{g}}$. Furthermore, based on thermodynamics techniques, 559 experimental data together with MD simulation blending are verified; thus, two 560 polymers PSF/PSMA thermodynamically compatible. In this perspective, membranes 561 such as for water treatment and fabrication of these blends (PSF and PSMA) can be 562 done via thermally induced phase separation method with glycol derivative diluent to 563 dissolve the polymer blends at high temperature. 


\section{Acknowledgment}

566 Authors gratefully acknowledge the Tianjin Science and Technology Planning Project

567 (Grant No. 18PTZWHZ00210), National Natural Science Foundation of China (Grant 568 No. 21576209 and 21878230), and Innovative Team in University of Ministry of

569 Education of China (Grant No. IRT-17R80) for their financial support. Also, I would

570 like to thank the School of Materials Science and Engineering, Tiangong University,

571 China, for their kind assistance.

*Declarations

574

575

Funding: (Authors gratefully acknowledge the Tianjin Science and Technology

576 Planning Project (Grant No. 18PTZWHZ00210), National Natural Science

577 Foundation of China (Grant No. 21576209 and 21878230), and Innovative Team in

578 University of Ministry of Education of China (Grant No. IRT-17R80)

579

580

Conflicts of interest/Competing interests: (No declared conflict of Interest)

581

582

Availability of data and material: (Not applicable)

583

584

Code availability: (Not applicable)

585

586

Authors' contributions: (Not applicable) 


\section{References}

[1] H. Abdul Mannan, T.M. Yih, R. Nasir, H. Muhktar, D.F. Mohshim, Fabrication and characterization of polyetherimide/polyvinyl acetate polymer blend membranes for $\mathrm{CO} 2 / \mathrm{CH} 4$ separation, Polymer Engineering \& Science 59(S1) (2019) E293-E301.

[2] M.R. Aguilar, J. San Román, PSMArt polymers, and their applications, Woodhead publishing2019.

[3] R.R. Luise, Applications of High-Temperature Polymers: 0, CRC Press2018.

[4] S.S. Jawalkar, K.V. Raju, S.B. Halligudi, M. Sairam, T.M. Aminabhavi, Molecular modeling simulations to predict the compatibility of poly (vinyl alcohol) and chitosan blends: a comparison with experiments, The Journal of Physical Chemistry B 111(10) (2007) 2431-2439.

[5] M. Linyu, W. Xigang, L. Yaqing, W. Junyuan, Computer Simulation of PAN/PVP Blends Compatibility and Preparation of Aligned PAN Porous Nanofibers via Magnetic-Field-Assisted Electrospinning PAN/PVP Blends, Materials Science 25(1) (2019) 54-59.

[6] J. Gupta, C. Nunes, S. Vyas, S. Jonnalagadda, Prediction of solubility parameters and miscibility of pharmaceutical compounds by molecular dynamics simulations, The Journal of Physical Chemistry B 115(9) (2011) 2014-2023.

[7] P. Barmpalexis, A. Karagianni, K. Katopodis, E. Vardaka, K. Kachrimanis, Molecular modeling and simulation of fusion-based amorphous drug dispersions in polymer/plasticizer blends, European Journal of Pharmaceutical Sciences 130 (2019) 260-268.

[8] B.D. Anderson, Predicting solubility/miscibility in amorphous dispersions: It is time to move beyond regular solution theories, Journal of pharmaceutical sciences 107(1) (2018) 24-33.

[9] I.M. de Arenaza, E. Meaurio, J.-R. Sarasua, Analysis of polymer blends' miscibility through molecular dynamics simulations, Polymerization (2012) 29. 
617

618

619

620

621

622

623

624

625

626

627

628

629

630

631

632

633

634

635

636

637

638

639

640

641

642

[10] S. Chang, X. Zhou, Z. Xing, Computing solubility parameters of phosphorus flame retardants by molecular dynamics and correlating their interactions with poly (ethylene terephthalate), Textile Research Journal 89(2) (2019) 195-203.

[11] M.B. Abderaman, E.-H.O. Gueye, A.N. Dione, A.A. Diouf, O. Faye, A.C. Beye, A Molecular Dynamics Study on the Miscibility of Polyglycolide with Different Polymers, International Journal of Materials Science and Applications 7(4) (2018) 126.

[12] C. Wu, W. Xu, Atomistic molecular simulations of structure and dynamics of crosslinked epoxy resin, Polymer 48(19) (2007) 5802-5812.

[13] M.S. Alam, J.H. Jeong, Thermodynamic properties and critical parameters of HFO-1123 and its binary blends with HFC-32 and HFC-134a using molecular simulations, International Journal of Refrigeration (2019).

[14] T.E. Gartner III, A. Jayaraman, Modeling and simulations of polymers: A Roadmap, Macromolecules 52(3) (2019) 755-786.

[15] T. Spyriouni, C. Vergelati, A molecular modeling study of binary blend compatibility of polyamide 6 and poly (vinyl acetate) with different degrees of hydrolysis: an atomistic and mesoscopic approach, Macromolecules 34(15) (2001) 5306-5316.

[16] C. Lavanya, K. Soontarapa, M. Jyothi, R.G. Balakrishna, Environmental friendly and cost-effective caramel for congo red removal, high flux, and fouling resistance of polysulfone membranes, Separation and Purification Technology 211 (2019) 348-358.

[17] W.E. Baker, C.E. Scott, G.-H. Hu, M. Akkapeddi, Reactive polymer blending, Hanser Munich2001.

[18] L.M. Robeson, Polymer blends, A Comprehensive Review (2007).

[19] M.T. DeMeuse, High-temperature polymer blends, Elsevier2014. 
644

645

646

647

648

649

650

651

652

653

654

655

656

657

658

659

660

661

662

663

664

665

666

667

668

669

670

671

[20] S. Shenvi, A. IPSMAil, A.M. Isloor, Enhanced permeation performance of cellulose acetate ultrafiltration membranes by incorporation of sulfonated poly (1, 4-phenylene ether sulfone) and poly (styrene-co-maleic anhydride), Industrial \& Engineering Chemistry Research 53(35) (2014) 13820-13827.

[21] P. Zhang, S. Xiang, H. Wang, Y. Wang, J. Zhang, Z. Cui, J. Li, B. He, Understanding the multiple functions of styrene-co-maleic anhydride in fabricating polyvinylidene fluoride hollow fiber membrane via coupled phase inversion process and its effect on surface infiltration behavior and membrane permeability, Journal of Membrane Science (2019) 117269.

[22] G.S. Ibrahim, A.M. Isloor, A.M. Asiri, A. IPSMAil, R. Kumar, M.I. Ahamed, Performance intensification of the polysulfone ultrafiltration membrane by blending with copolymer encompassing novel derivative of poly (styrene-comaleic anhydride) for heavy metal removal from wastewater, Chemical Engineering Journal 353 (2018) 425-435.

[23] S.L. Mayo, B.D. Olafson, W.A. Goddard, DREIDING: a generic force field for molecular simulations, Journal of Physical chemistry 94(26) (1990) 8897-8909.

[24] H. Meirovitch, Computer simulation of self-avoiding walks: Testing the scanning method, The Journal of chemical physics 79(1) (1983) 502-508.

[25] D.N. Theodorou, U.W. Suter, Detailed molecular structure of a vinyl polymer glass, Macromolecules 18(7) (1985) 1467-1478.

[26] D. Ongari, P.G. Boyd, O. Kadioglu, A.K. Mace, S. Keskin, B. Smit, Evaluating charge equilibration methods to generate electrostatic fields in nanoporous materials, Journal of chemical theory and computation 15(1) (2018) 382-401.

[27] X. Wu, B.R. Brooks, The homogeneity condition: A simple way to derive isotropic periodic sum potentials for efficient calculation of long-range interactions in molecular simulation, The Journal of chemical physics 150(21) (2019) 214109.

[28] C.M. Hansen, Hansen solubility parameters: a user's handbook, CRC press2002. 
672 [29] M. Zhang, P. Choi, U. Sundararaj, Molecular dynamics and thermal analysis 673 study of the anomalous thermodynamic behavior of poly (ether 674 imide)/polycarbonate blends, Polymer 44(6) (2003) 1979-1986.

675 [30] J.E. Mark, Polymer data handbook, (2009).

676 [31] C. Li, A. Strachan, Cohesive energy density and solubility parameter evolution 677 during thermoset curing, Polymer 135 (2018) 162-170.

678 [32] D. Paul, J. Barlow, H. Keskkula, Mark-Bikales-Overberger-Menges: 679 Encyclopedia of Polymer Science and Engineering, Vol. 12, John Wiley, New $680 \quad$ York, 1988.

681 [33] S. Li, Z. Cui, L. Zhang, B. He, J. Li, The effect of sulfonated polysulfone on the 682 compatibility and structure of polyethersulfone-based blend membranes, Journal 683 of membrane science 513 (2016) 1-11.

684 [34] D.R. Paul, Polymer blends, Elsevier2012.

685 [35] R. Fedors, D. Van Krevelen, P. Hoftyzer, C. In, Handbook of solubility 686 687 parameters and other cohesion parameters, Barton, AFM, CRC Press: Boca Raton, FL, 1983.

688

[36] G. Brannock, J. Barlow, D.R. Paul, Blends of styrene/maleic anhydride 689 copolymers with polymethacrylates, Journal of Polymer Science Part B: Polymer 690 Physics 29(4) (1991) 413-429.

691

[37] A. Shulkin, H.D. Stöver, Microcapsules from styrene-maleic anhydride 692 693 copolymers: the study of morphology and release behavior, Journal of membrane science 209(2) (2002) 433-444.

694 [38] A. Shulkin, Styrene-Maleic Anhydride, and Styrene-Ma1eimide Based 695 Copolymers as Building Blocks in Microencapsulation Procedures, 2002.

696 [39] Y. Aoki, Miscibility of poly (acrylonitrile-co-styrene) with poly [styrene-co697 (maleic anhydride)] and poly [styrene-co-(N-phenyl maleimide)], 698 Macromolecules 21(5) (1988) 1277-1282. 
[40] K.S. Schweizer, J.G. Curro, Integral equation theory of the structure and thermodynamics of polymer blends, The Journal of Chemical Physics 91(8) (1989) 5059-5081.

[41] J.G. Curro, K.S. Schweizer, G.S. Grest, K. Kremer, A comparison between integral equation theory and molecular dynamics simulations of dense, flexible polymer liquids, The Journal of Chemical Physics 91(2) (1989) 1357-1364.

[42] K.F. Freed, New lattice model for interacting, avoiding polymers with controlled length distribution, Journal of Physics A: Mathematical and General 18(5) (1985) 871.

[43] C.F. Fan, B.D. Olafson, M. Blanco, S.L. Hsu, Application of molecular simulation to derive phase diagrams of binary mixtures, Macromolecules 25(14) (1992) 3667-3676.

[44] F. Case, J. Honeycutt, Will my Polymers Mix?-Applications of Modelling to Study Miscibility, Compatibility, and Formulation, Trends in Polymer Science 2(8) (1994) 259-266.

[45] M. Amini, M. Mobli, M. Khalili, H. Ebadi-Dehaghani, Assessment of compatibility in Polypropylene/Poly (lactic acid)/Ethylene vinyl alcohol ternary blends: relating experiments and molecular dynamics simulation results, Journal of Macromolecular Science, Part B 57(4) (2018) 287-304.

[46] S.S. Jawalkar, S.G. Adoor, M. Sairam, M.N. Nadagouda, T.M. Aminabhavi, Molecular modeling on the binary blend compatibility of poly (vinyl alcohol) and poly (methyl methacrylate): an atomistic simulation and thermodynamic approach, The Journal of Physical Chemistry B 109(32) (2005) 15611-15620.

[47] L. Feizhou, Z.-L. Lu, X.-s. Wang, Y.-t. Xi, A combined experimental and molecular dynamics simulation study on Eucommia ulmoides gum's miscibility with butadiene rubber, Journal of Polymer Research 24(7) (2017) 100.

[48] F.-Z. Li, Z.-L. Lu, D.-P. Tian, A Combined Experimental and Molecular Dynamics Simulation Study on Eucommia Ulmoides Gum's Miscibility with Several Rubbers, Polymers and Polymer Composites 25(1) (2017) 87-92. 
[49] P.H. Brodsky, COMPATIBILITY IN POLYMER BLENDS, (1970).

[50] L.A. Utracki, B. Favis, Polymer alloys and blends, Handbook of polymer science and technology 4 (1989) 121-185.

[51] J. Barlow, D.R. Paul, Polymer blends and alloys-a review of selected considerations, Polymer Engineering \& Science 21(15) (1981) 985-996.

[52] J.C. Mitchell, J.L. French, Analytical crucible, Google Patents, 2001.

[53] M. Padaki, A.M. Isloor, G. Belavadi, K.N. Prabhu, Preparation, characterization, and performance study of poly (isobutylene-alt-maleic anhydride)[PIAM] and polysulfone [PSf] composite membranes before and after alkali treatment, Industrial \& Engineering Chemistry Research 50(11) (2011) 6528-6534.

[54] J. Yoo, J. Kim, Y. Kim, C. Kim, Novel ultrafiltration membranes prepared from the new miscible blends of polysulfone with poly (1-vinylpyrrolidone-co-styrene) copolymers, Journal of membrane science 216(1-2) (2003) 95-106.

[55] Y. Wang, H. Song, H. Ge, J. Wang, Y. Wang, S. Jia, T. Deng, X. Hou, Controllable degradation of polyurethane elastomer via selective cleavage of $\mathrm{CO}$ and CN bonds, Journal of cleaner production 176 (2018) 873-879.

[56] T.R. Crompton, Thermal methods of polymer analysis, Smithers Rapra2013.

[57] J. Baker, An algorithm for the location of transition states, Journal of Computational Chemistry 7(4) (1986) 385-395.

[58] K. Pajula, M. Taskinen, V.-P. Lehto, J. Ketolainen, O. Korhonen, Predicting the formation and stability of amorphous PSMAll molecule binary mixtures from computationally determined Flory- Huggins interaction parameter and phase diagram, Molecular Pharmaceutics 7(3) (2010) 795-804.

[59] N. Wang, X. Huang, H. Gong, Y. Zhou, X. Li, F. Li, Y. Bao, C. Xie, Z. Wang, Q. Yin, Thermodynamic mechanism of selective cocrystallization explored by MD simulation and phase diagram analysis, AIChE Journal 65(5) (2019).

[60] B. Schneier, Polymer compatibility, Journal of Applied Polymer Science 17(10) (1973) 3175-3185. 


\section{Supplementary Files}

This is a list of supplementary files associated with this preprint. Click to download.

- supplementaryfigures.docx 